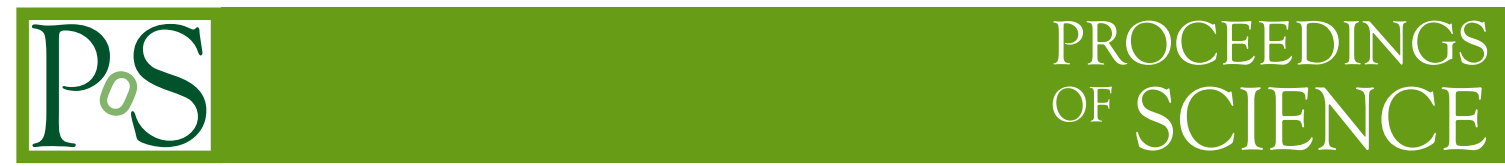

\title{
The Non-existence of the Proton Spin Crisis
}

\author{
Elliot Leader* \\ Imperial College London \\ e.leadereimperial.ac.uk
}

The spin crisis arose some 30 years ago following results of a European Muon Collaboration experiment on polarized DIS. Since then many people have pointed out that the arguments involved are too naive, yet papers continue to appear devoted to "the spin crisis". We explain the subtleties involved in giving a precise statement of the spin sum rule and clarify why there is really no crisis.

23rd International Spin Physics Symposium - SPIN2018 -

10-14 September, 2018

Ferrara, Italy

${ }^{*}$ Speaker. 
The controversy began some decades ago when the European Muon Collaboration (EMC) published results of their experiment on polarized fully inclusive DIS, which suggested that the constituents of the proton could not provide enough angular momentum to explain the fact that the proton had spin 1/2. And so 30 years ago Mauro Anselmino and E.L. published a paper in response to the results of the EMC experiment entitled A crisis in the parton model: Where, oh where is the proton's spin?[1].

However, it has long been understood that there is no such crisis. Nonetheless papers keep appearing referring to "The proton spin crisis" . Why??????

Because people have forgotten that the belief in a spin-crisis emerged from an over naive interpretation of the EMC experiment. (For a modern summary of the situation and access to the literature, see the review of Kuhn, Chen and Leader [2]).

\section{The naive interpretation of the EMC results}

The Quark Model of the nucleon had offered a fairly successful description of the nucleon and the baryon resonances:

$$
\text { Nucleon }=\text { bound state of } 3 \text { massive quarks }(Q) \quad\left(M_{Q} \approx M_{N} / 3\right)
$$

The nucleon corresponds to the ground state, and for any reasonably behaved potential this will be an s-state. In the simplest non-relativistic case, for the constituents in an s-state, the quarks have no OAM (orbital angular momentum). Hence, for a nucleon at rest, say polarized in the positive Z-direction

$$
1 / 2=S_{z}^{N}=\sum_{Q} S_{z}^{Q}
$$

\section{Theoretical interpretation of the EMC polarized DIS experimental results}

The quantity of interest is the first moment of the spin-dependent structure function $g_{1}\left(x, Q^{2}\right)$

$$
\Gamma_{1}^{p} \equiv \int_{0}^{1} g_{1}^{p}(x) d x
$$

In NLO the expression for $\Gamma_{1}^{p}$ is renormalization scheme dependent. In the $\overline{M S}$ scheme, at leading twist, valid for $Q^{2} \gg M^{2}$,

$$
\Gamma_{1}^{p}\left(Q^{2}\right)=\frac{1}{12}\left[\left(a_{3}+\frac{1}{3} a_{8}\right) \Delta C_{N S}^{\overline{M S}}+\frac{4}{3} a_{0}\left(Q^{2}\right) \Delta C_{S}^{\overline{M S}}\right],
$$

where the $\Delta C$ are the known singlet and non-singlet Wilson coefficients.

$a_{3,8}$ are matrix elements of non-singlet SU(3) currents and $a_{0}$ of the singlet SU(3) current. Now the values of $a_{3,8}$ can be obtained from neutron and hyperon $\beta$-decay. Hence a measurement of $\Gamma_{1}^{p}$ at some value of $Q^{2}$ is effectively a measure of $a_{0}\left(Q^{2}\right)$, where the $Q^{2}$ dependence of $a_{0}$ arises because the singlet current has to be renormalized, and it is customary and convenient to choose $Q^{2}$ as the 
renormalization scale.

Now

$$
\begin{aligned}
a_{0} & =\Delta \Sigma \equiv(\Delta u+\Delta \bar{u})+(\Delta d+\Delta \bar{d})+(\Delta s+\Delta \bar{s}) \\
& =2\left[\sum_{q}\left\langle S_{z}^{q}\right\rangle+\sum_{\bar{q}}\left\langle S_{z}^{\bar{q}}\right\rangle\right]
\end{aligned}
$$

Hence if there is no other source of angular momentum one expects

$$
\left[\sum_{q}\left\langle S_{z}^{q}\right\rangle+\sum_{\bar{q}}\left\langle S_{z}^{\bar{q}}\right\rangle\right]=S_{z}^{\text {proton }}=1 / 2
$$

implying, naively,

$$
a_{0}=1
$$

. The EMC experiment gave $a_{0} \approx 0$ and later experiments confirmed that $a_{0} \ll 1$, giving rise to the spin crisis in the (naive) parton model. However,

$$
a_{0}=1
$$

cannot possibly be true because the right hand side is a fixed number, whereas the left hand side is, beyond the naive level, equal to $a_{0}\left(Q^{2}\right)$, i.e. a function of $Q^{2}$ ! Thus failure of Eq. (??) to hold cannot, in principle, be used to infer that there is a spin crisis.

\section{The Non-naive interpretation}

Clearly a correct relation between the spin of a nucleon and the angular momentum of its constituents should include their OAM and a contribution from the gluons. Unfortunately this is much more complicated than it sounds, because there is some controversy as to which operators should be used to represent the angular momentum, especially in the case of the massless gluon. (See [3] and the review by Leader and Lorcé [4]).

\section{Expression based on the canonical (can) version of the angular momentum}

The sum rule relating the angular momentum (AM) of its constituents to the AM of the proton

$$
\frac{1}{2}=\left\langle\left\langle\hat{S}_{z}^{q}\right\rangle\right\rangle+\left\langle\left\langle\hat{L}_{z}^{q}\right\rangle\right\rangle+\left\langle\left\langle\hat{S}_{z}^{G}\right\rangle\right\rangle+\left\langle\left\langle\hat{L}_{z}^{G}\right\rangle\right\rangle
$$

looks totally intuitive; can't be incorrect!

It is usually written in the Jaffe-Manohar form :

$$
\frac{1}{2}=\frac{1}{2} a_{0}+\Delta G+\left\langle\left\langle\hat{L}_{z}^{q}\right\rangle\right\rangle+\left\langle\left\langle\hat{L}_{z}^{G}\right\rangle\right\rangle
$$

but more correctly it should read :

$$
\frac{1}{2}=\frac{1}{2} a_{0}+\Delta G+\left\langle\left\langle\hat{L}_{\mathrm{can}, z}^{q}\right\rangle\right\rangle+\left\langle\left\langle\hat{L}_{\mathrm{can}, z}^{G}\right\rangle\right\rangle .
$$


But is the Jaffe-Manohar form really identical to the original canonical form??? DANGER! $\triangle G$ is a gauge invariant quantity but $\left\langle\left\langle S_{\mathrm{can}, z}^{G}\right\rangle\right\rangle$ is not.

However one can show that

$$
\Delta G=\left.\left\langle\left\langle\hat{S}_{\text {can }, z}^{G}\right\rangle\right\rangle\right|_{\text {Gauge } A^{0}=0},
$$

or, as the nucleon momentum $P \rightarrow \infty$

$$
\Delta G=\left\langle\left.\left\langle\hat{S}_{\text {can }, z}^{G}\right\rangle\right|_{\text {Gauge } A^{+}=0} .\right.
$$

Moreover the operators $\hat{L}^{q, G}$ are also not gauge invariant. Thus all the gauge non-invariant operators appearing in the Jaffe-Manohar sum rule should be evaluated in the gauge $A^{0}=0$ or $A^{+}=0$.

\section{Rigorous statement of AM sum rule}

Finally for a fast moving proton with helicity $+1 / 2$ the angular momentum sum rule becomes, in contrast to the naive result

$$
\frac{1}{2}=\frac{1}{2} a_{0}+\Delta G+\left.\left\langle\left\langle\hat{L}_{\text {can }, z}^{q}\right\rangle\right\rangle\right|_{A^{+}=0}+\left.\left\langle\left\langle\hat{L}_{\text {can }, z}^{G}\right\rangle\right\rangle\right|_{A^{+}=0} .
$$

It should not be forgotten that each individual term in is actually a function of $Q^{2}$, but that the sum is not.

Now $\Delta G$ can be measured and seems to be relatively small, but not negligible, typically $\Delta G \approx$ $0.29 \pm 0.32$ for $Q^{2} \approx 10 G e V^{2}$. There are suggestions about how to measure the orbital AM terms, but as yet there are no experimental results. So the challenge is to find a way to measure the orbital terms and thus to check whether the sum rule holds. Only then will one be able to claim that there is or is not a spin crisis.

\section{A different version of the sum rule}

All of the above utilized the canonical version of the angular momentum operators. There is also a perfectly good sum rule based on the Belinfante version of the operators:

$$
\frac{1}{2}=\left\langle\left\langle\hat{S}_{z}^{q}\right\rangle\right\rangle+\left\langle\left\langle\hat{L}_{\mathrm{Bel}, z}^{q}\right\rangle\right\rangle+\left\langle\left\langle\hat{J}_{\mathrm{Bel}, z}^{G}\right\rangle\right\rangle
$$

In this case the total AM of the gluons is not split into spin and orbital parts. Also, here, the operators are gauge invariant and can thus be studied relatively simply on a lattice. Beautiful results [5] indicate significant contributions to the proton spin from the gluons (28\%) and from the orbital AM of the up, down and strange quarks (48\%). So, in the Belinfante version, where the contributions from the constituents can at least be estimated via lattice calculations, there is no hint of any sort of crisis.

\section{Conclusions}

What appeared to be a spin crisis in the parton model, 30 years ago, was a consequence of a misinterpretation of the results of the European Muon Collaboration experiment on polarized deep 
inelastic scattering. This was caused by a failure to distinguish adequately between constituent and partonic quarks. In constituent quark models the spin of the nucleon is built up largely from the spins of its constituents.

This is not true for partonic quarks, and the smallness of the spin contribution of the partonic quarks is not unreasonable, given that they certainly possess orbital angular momentum as well, and that the gluons, too, carry some angular momentum. In the usual canonical form of the spin sum rule it is known from experiment that the gluon spin is non-negligible, but we do not yet have experimental results about the quark and gluon orbital angular momentum, so that there is absolutely no basis for the assertion that there is a nucleon spin crisis.

Moreover an alternative version of the spin sum rule based on Belinfante operators, where all terms can be measured on a lattice, likewise, shows absolutely no signs of a crisis.

\section{References}

[1] E. Leader and M. Anselmino, A CRISIS IN THE PARTON MODEL: WHERE, OH WHERE IS THE PROTON'S SPIN?, Z.Phys. C41 (1988) 239.

[2] S. E. Kuhn, J. P. Chen and E. Leader, Spin Structure of the Nucleon - Status and Recent Results, Prog. Part. Nucl. Phys. 63 (2009) 1 [0812.3535].

[3] E. Leader, On the controversy concerning the definition of quark and gluon angular momentum, Phys.Rev. D83 (2011) 096012 [1101.5956].

[4] E. Leader and C. Lorce, The angular momentum controversy: What's it all about and does it matter?, Phys.Rept. (2013) [1309.4235].

[5] K. Liu, M. Deka, T. Doi, Y. Yang, B. Chakraborty et al., Quark and Glue Momenta and Angular Momenta in the Proton - a Lattice Calculation, PoS LATTICE2011 (2011) 164 [1203. 6388]. 